\title{
Finite time vacuum survival amplitude and vacuum energy decay
}

\author{
Enrique Álvarez* ${ }^{*}$ and Roberto Vidal ${ }^{\dagger}$ \\ Instituto de Física Teórica UAM/CSIC and Departamento de Física Teórica, Universidad Autónoma de Madrid, \\ E-28049-Madrid, Spain
}

(Received 12 August 2011; published 8 November 2011; publisher error corrected 10 November 2011)

\begin{abstract}
The problem of the vacuum energy decay is studied for both signs of the cosmological constant, through the analysis of the vacuum survival amplitude, defined in terms of the conformal time, $z$, by $\mathcal{A}\left(z, z^{\prime}\right) \equiv$ $\left\langle\operatorname{vac} z \mid \operatorname{vac} z^{\prime}\right\rangle$. Transition amplitudes are computed for finite time span, $Z \equiv z^{\prime}-z$, and their late time behavior (directly related to the putative decay width of the state), and the transients are discussed up to first order in the coupling constant, $\lambda$.
\end{abstract}

DOI: $10.1103 /$ PhysRevD.84.105009

PACS numbers: 11.10.Kk, 04.62.+v

\section{INTRODUCTION}

It has been claimed [1] that the free energy corresponding to an interacting theory in de Sitter space has got an imaginary part that can be interpreted as some sort of instability.

The way this imaginary part has to be (perhaps naively) computed is by doing the path integral at imaginary time. In Poincaré coordinates of de Sitter space $\left(d S_{n}\right)$ in which the metric reads

$$
d s^{2}=\frac{l^{2} d z^{2}-\sum_{i}\left(d x^{i}\right)^{2}}{z^{2}}
$$

this leads to the metric of Euclidean (anti) de Sitter space $\left(E A d S_{n}\right)$ with metric

$$
d s^{2}=\frac{l^{2} d z^{2}+\sum_{i}\left(d x^{i}\right)^{2}}{z^{2}}
$$

(and not to the metric on the sphere $S_{n}$ ), and the corresponding free energy has been computed by us up to the one-loop order in the first paper of [2], where no imaginary part was find to that order.

Some general arguments can be advanced, however, supporting the idea that a nonvanishing result should be found to higher-loop order. Namely, in the second paper of [2], it was pointed out that (if it were applicable) the optical theorem relates the (technically quite difficult) computation of the imaginary part of the free energy to a much simpler tree level calculation, i.e., the vacuum decay into identical particles [3]. A related phenomenon is the decay of a particle into several identical particles [4]. Besides, there is no reason for this effect to be restricted to de Sitter space; on the contrary, it would be natural to expect it to be quite generic.

It is true, however, that all our intuition is based upon flat space examples, with the ensuing asymptotic regions, and S-matrix elements that can be computed through Lehmann-Symanzik-Zimmermann techniques [5]. Outside

\footnotetext{
*enrique.alvarez@uam.es

†jroberto.vidal@uam.es
}

this framework, it is not even known how to define a particle to be decayed into nor the interacting vacuum $|\mathrm{vac}\rangle$ in the absence of a well-defined energetic argument.

A related issue is the study of the time dependence of transition amplitudes. The linear dependence in time is one of the key aspects of Fermi's golden rule. The fact that is problematic in curved space, where there is no naturally preferred coordinate system in general, has been remarked in [4]. It is to be stressed that use of non-Cartesian coordinates is not without problems even in flat space-time, and this is even more true about polar coordinates in field space. One of the purposes of the present work is to examine this problem, by computing overlaps between states that differ by a finite time in whatever coordinate system we are using.

The paper is organized as follows. In Sec. II we review some results regarding transition amplitudes in both flat and curved space-time, and we present the basic quantummechanical formalism that we will use in our calculations. It is based on the functional Schrödinger picture for finite time intervals. In Sec. III, we put our techniques to work in order to recover the standard quantum evolution in Minkowski space-time. This we do in order to check our formalism, and to compare with ordinary quantum mechanics (as opposed to field theory). In Sec. IV we apply this formalism to de Sitter (and briefly to anti-de Sitter) to examine the (conformal time) dependence of its transition amplitudes. Some technical details have been relegated to the appendixes.

\section{OVERLAPS AND EVOLUTION}

In flat $n$-dimensional space-time [5] (where energy conservation holds) differential transition amplitudes from an initial state with $N_{i}$ initial particles do behave for $T$ large enough as

$$
d \Gamma \sim T V_{n-1}^{1-N_{i}}
$$

where $T$ is the time span during which the interaction is turned on, and $V_{n-1}$ is the volume of the codimension-1 spatial sections of constant time of the system (so that in 
the particular case of vacuum decay it is proportional to the full $n$-dimensional volume).

In the opposite limit, Maiani and Testa [6] have shown that there is a divergence at small times $T \rightarrow 0$ which survives even after renormalization. In order to eliminate it, a careful study of the incoming wave packet is necessary. They studied, in particular, the example of an unstable scalar particle of mass $M$ decaying into two other scalar particles with masses $m_{1}$ and $m_{2}$. In the narrow packet approximation for the initial state they were able to prove that

$$
S(t)=2 \pi g^{2} e^{-i M t}\left(\alpha_{0}+\alpha_{1}+\beta_{1}(t)\right)
$$

where $M$ is the renormalized mass of the resonant state.

The assumption will be made in Sec. that the quantummechanical formula

$$
\left\langle\varphi_{f} t_{f} \mid \varphi_{i} t_{i}\right\rangle \equiv\left\langle\varphi_{f}\left|e^{-i H\left(t^{\prime}-t\right)}\right| \varphi_{i}\right\rangle \equiv \int_{\varphi_{i}}^{\varphi_{f}} \mathcal{D} \phi e^{i S[\phi]}
$$

remains valid in curved backgrounds, where the Hamiltonian is generically time-dependent.

\section{Survival amplitudes}

Let us introduce the general formalism first in flat space language, but in such a way that it is easily amenable to generalizations to curved space. The whole aim of the present work is to compute the overlap between an state |in $\rangle$ defined at a given time $t_{i}$ and another state |out〉 defined at a different time $t_{f}$ (both times can be finite). These would become S-matrix elements in case $t_{i} \rightarrow-\infty$, $t_{f} \rightarrow \infty$ and the interaction (including the one resulting from the background gravitational field, if any) is assumed to be switched off at asymptotic times.

First of all, the survival amplitude is an overlap

$$
\mathcal{A}\left(t_{f}, t_{i}\right) \equiv\left\langle\psi\left(t_{f}\right) \mid \psi\left(t_{i}\right)\right\rangle
$$

If the space is normalized so that

$$
\left\langle\psi\left(t_{i}\right) \mid \psi\left(t_{i}\right)\right\rangle=1
$$

then the unitary evolution (this is a crucial hypothesis) does preserve the norm, so that

$$
\left\langle\psi\left(t_{f}\right) \mid \psi\left(t_{f}\right)\right\rangle=1 .
$$

Then Cauchy-Schwarz's inequality guarantees that

$$
\begin{aligned}
\left|\mathcal{A}\left(t_{f}, t_{i}\right)\right| & \equiv\left|\left\langle\psi\left(t_{f}\right) \mid \psi\left(t_{i}\right)\right\rangle\right| \\
& \leq\left|\left\langle\psi\left(t_{i}\right) \mid \psi\left(t_{i}\right)\right\rangle\right| \cdot\left|\left\langle\psi\left(t_{f}\right) \mid \psi\left(t_{f}\right)\right\rangle\right|=1 .
\end{aligned}
$$

This means that the quantity $\left(T \equiv t_{f}-t_{i}\right)$

$$
\Gamma(T) \equiv-\frac{2}{T} \log \left|\mathcal{A}\left(t_{f}, t_{i}\right)\right|
$$

must be positive, and in case it is independent of $T$ in the asymptotic regime, could be rightfully interpreted as the decay width of the state. We shall refer to it loosely as decay width even when it is not constant.

Survival amplitudes are therefore powerful tools to detect instabilities; they are, however, somewhat blind to the final state of such decays; we will have no precise information on the decay products. This appears to be an important open problem from this viewpoint.

The first principles path integral formula reads

$$
\begin{aligned}
\left.\mathcal{S}\left(t_{f}, t_{i}\right)\right|_{J} \equiv & \langle\text { out }| \text { in }\rangle\left.\right|_{J} \\
= & \int\left[D \varphi_{f}\right]\left[D \varphi_{i}\right] \Psi_{t_{f}}\left[\varphi_{f}\right]^{*} \\
& \times\left.\left\langle\varphi_{f} t_{f} \mid \varphi_{i} t_{i}\right\rangle\right|_{J} \Psi_{t_{i}}\left[\varphi_{i}\right],
\end{aligned}
$$

where the integration measure $[D \varphi]$ is defined in the space of field configurations at fixed "time." The wave functionals, which are functionals of this fields, are given by

$$
\left.\left.\Psi_{t_{f}}\left[\varphi_{f}\right] \equiv\left\langle\varphi_{f} t_{f}\right| \text { out }\right\rangle \quad \Psi_{t_{i}}\left[\varphi_{i}\right] \equiv\left\langle\varphi_{i} t_{i}\right| \text { in }\right\rangle .
$$

An external source $J$ is introduced as usual in order to treat interactions by functional differentiation.

The problem is then reduced to first computing the wave functionals of both states (itself a nontrivial task), and then the field transition amplitude, which is really the Feynman Kernel, or in modern parlance essentially the Schrodinger functional

$$
\left.K[J]\left[\varphi_{f} t_{f}, \varphi_{i} t_{i}\right] \equiv\left\langle\varphi_{f} t_{f} \mid \varphi_{i} t_{i}\right\rangle\right|_{J}
$$

followed by a final functional integration over the possible values of the fields.

This Schrodinger functional will be computed using the general expression in terms of path integral

$$
\begin{aligned}
K[J]\left[\varphi^{\prime} t^{\prime}, \varphi t\right] & =\left\langle\varphi^{\prime} t^{\prime} \mid \varphi t\right\rangle \\
& \equiv \int_{\phi(\cdot, t)=\varphi}^{\phi\left(\cdot t^{\prime}\right)=\varphi^{\prime}} \mathcal{D} \phi e^{i \int_{\Sigma_{t}}^{\Sigma_{t^{\prime}} d^{n} x L(\phi, \partial \phi)},}
\end{aligned}
$$

where $\Sigma_{t}$ is a codimension-1 hypersurface of constant time.

Let us remind ourselves of some well-known facts in quantum mechanics (cf., for example, [7]). If the initial state $\mid$ in, $\left.t_{i}\right\rangle$ (where we have explicitly indicated the possible time dependence), be it the vacuum state or otherwise, is an eigenstate of the full Hamiltonian, then Schrödinger's equation imply that the modulus of the survival rate is equal to one and this is true for any values of $t_{i}$ and $t_{f}$ :

$$
\left.\left|\mathcal{A}_{\text {in }}\left(t_{f}, t_{i}\right)\right| \equiv \mid\left\langle\text { in } t_{f}\right| \text { in } t_{i}\right\rangle \mid=1 .
$$

It is plain that the survival rate is nothing else than the selfoverlap $(\mid$ in $\rangle=\mid$ out $\rangle)$ at finite time interval.

This means that the only way a state (vacuum or otherwise) can be unstable is by it being a superposition of energy eigenstates. Then the study of the survival amplitude for finite time is quite useful, because we do not need 
to know any details of the decay process (which is a complicated thing in the absence of asymptotically flat regions).

In order for a given state to be unstable it is not enough that the survival rate depends on time (this happens already for a linear superposition of only two energy eigenstates), but that this dependence has to be monotonic in time. It is enough, for example, that

$$
\dot{\mathcal{A}} \neq 0, \quad \forall t .
$$

The actual dependence of the survival rate in quantum field theory with the time interval is however quite complicated. Besides the divergence at small times uncovered by Maiani and Testa [6] (whose understanding demands a careful treatment of wave packets in the initial state), it can be explicitly shown in some models that the behavior is oscillating, except at asymptotic times $\left(T \equiv t_{f}-t_{i} \rightarrow \infty\right)$.

\section{SURVIVAL AMPLITUDES IN FLAT SPACE}

It has been already advertised that the use of our techniques is best illustrated in the simplest flat space example. It is going to be a rather long computation, so let us now draw its road map. There are three steps. The most important one is the computation of Schrödinger functional or Feynman kernel; which is the quantum-mechanical transition amplitude between states with well-defined values for the fields. This involves the computation of a determinant with Dirichlet boundary conditions. The final step is to integrate over the boundary values of the fields, weighted by the wave functional of the state, which we also need to know at this stage.

\section{A. Wave functionals}

This means that the first thing we have to do is to find the wave functionals $[8,9]$ of the states $\mid$ in $\rangle$ and $\mid$ out $\rangle$. Let us begin with the vacuum. First of all, in flat space the free field and momentum operators read ${ }^{1}$

$$
\begin{aligned}
\phi(\vec{x}) & =\int \frac{d \vec{p}}{(2 \pi)^{(n-1) / 2}} \frac{1}{\sqrt{2 \omega_{p}}}\left(a_{\vec{p}} e^{i \vec{p} \vec{x}}+a_{\vec{p}}^{\dagger} e^{-i \vec{p} \vec{x}}\right) \pi(\vec{x}) \\
& =-i \int \frac{d \vec{p}}{(2 \pi)^{(n-1) / 2}} \sqrt{\frac{\omega_{p}}{2}}\left(a_{\vec{p}} e^{i \vec{p} \vec{x}}-a_{\vec{p}}^{\dagger} e^{-i \vec{p} \vec{x}}\right)
\end{aligned}
$$

so that

$$
a_{\vec{p}}=\frac{1}{\sqrt{2}} \int \frac{d \vec{x}}{(2 \pi)^{(n-1) / 2}} e^{-i \vec{p} \vec{x}}\left(\sqrt{\omega_{p}} \phi(\vec{x})+i \frac{1}{\sqrt{\omega_{p}}} \pi(\vec{x})\right)
$$

in such a way that the vacuum wave functional obeys

$$
\int \frac{d \vec{x}}{(2 \pi)^{(n-1) / 2}} e^{i \vec{p} \vec{x}}\left(\sqrt{\omega_{p}} \varphi(\vec{x})+\sqrt{\frac{1}{\omega_{p}}} \frac{\delta}{\delta \varphi(\vec{x})}\right)\langle\varphi \mid 0\rangle=0
$$

\footnotetext{
${ }^{1}$ We will work in $n$ dimensions, so the vector notation $\vec{x}, \vec{p}$, etc. will mean always an integration over $n-1$ variables.
}

so that the solution looks exactly the same as in $[8,9]$, namely,

$$
\Psi_{0}[\varphi] \equiv\langle\varphi \mid 0\rangle=N e^{-(1 / 2) \int d \vec{x} d \vec{y} \omega(\vec{x}, \vec{y}) \varphi(\vec{x}) \varphi(\vec{y})}
$$

with

$$
\omega(\vec{x}, \vec{y})=\omega(\vec{y}, \vec{x})=\int \frac{d \vec{k}}{(2 \pi)^{n-1}} e^{i \vec{k}(\vec{x}-\vec{y})} \omega_{k} .
$$

Using the functional Schrödinger's equation

$$
\begin{aligned}
& i \frac{\partial}{\partial t} \Psi_{0}[\varphi, t] \\
& \quad=\frac{1}{2} \int d \vec{x}\left(-\frac{\delta^{2}}{\delta \varphi^{2}}-\eta^{i j} \partial_{i} \varphi \partial_{j} \varphi+m^{2} \varphi^{2}\right) \Psi_{0}[\varphi, t]
\end{aligned}
$$

it is possible to determine

$$
\Psi[\varphi, t]=N e^{-i E_{0} t} \Psi[\varphi]
$$

with the vacuum energy defined as

$$
\begin{aligned}
E_{0} & \equiv \frac{1}{2} \operatorname{tr} \omega \equiv \frac{1}{2} \int d \vec{x} \omega(\vec{x}, \vec{x})=V_{n-1} \rho_{0} \\
& =\frac{V_{n-1}}{2} \int \frac{d \vec{k}}{(2 \pi)^{n-1}} \omega_{k}
\end{aligned}
$$

where the spatial volume is denoted by $V_{n-1} \equiv \int d \vec{x}$. From the expression above it is plain that $E_{0}$ is both ultravioletand infrared-divergent.

The time-independent normalization factor is given by

$$
N=\operatorname{det}\left(\frac{\omega}{\pi}\right)^{1 / 4}=e^{V_{n-1} / 4 \int\left(d \vec{k} /(2 \pi)^{n-1}\right) \log \left(\omega_{k} / \pi\right)} .
$$

It is useful to consider eigenfunctions of the kernel defined such that

$$
\int d \vec{y} \omega(\vec{x}, \vec{y}) f_{\epsilon}(\vec{y}) \equiv \epsilon f_{\epsilon}(\vec{x}) .
$$

In flat space those are just plane waves

$$
f_{\epsilon}(\vec{x}) \equiv e^{i \vec{p} \vec{x}}
$$

and the eigenvalue reads

$$
\epsilon \equiv \omega_{p} .
$$

In momentum space the vacuum wave functional reads

$$
\Psi_{0}[\phi]=e^{-(1 / 2) \int\left(d \vec{k} /(2 \pi)^{n-1}\right) \omega_{k} \phi_{-k} \phi_{k}}
$$

where

$$
\phi_{k} \equiv \int d \vec{x} e^{-i \vec{k} \vec{x}} \phi_{\vec{x}}
$$

\section{B. Inclusion of the interaction}

First we write as usual the interacting kernel in terms of the free one, using sources 


$$
K[J]=e^{i \int_{t_{i}}^{t_{f}} d t d \vec{x} L_{I}(\delta / i \delta J)} K_{0}[J],
$$

where $K_{0}$ is the kernel that corresponds to the free action

$$
\begin{aligned}
S_{0}[\phi]-J \phi \equiv & \int d^{n} x\left(\frac{1}{2}(\partial \phi)^{2}-\frac{m^{2}}{2} \phi^{2}-J(x) \phi(x)\right) \\
= & \int \frac{d \vec{k}}{(2 \pi)^{n-1}} \int_{t_{i}}^{t_{f}} d t\left(\frac{1}{2}\left|\dot{\phi}_{k}\right|^{2}-\frac{\omega_{k}^{2}}{2}\left|\phi_{k}\right|^{2}\right. \\
& \left.-j_{-k}(t) \phi_{k}(t)\right) .
\end{aligned}
$$

In order to perform the functional integration, we follow Sakita [10] and split the field into a classical piece, $\phi_{k}^{c}(t)$ (with boundary conditions yet to be specified) and a quantum part, $\chi_{k}(t)$

$$
\phi_{k}(t)=\phi_{k}^{c}(t)+\chi_{k}(t)
$$

so that we have for the measure $\mathcal{D} \phi=\mathcal{D} \chi$, as well as

$$
\begin{aligned}
& S_{0}[\phi]-J \phi \\
& =S_{0}\left[\phi^{c}\right]+S_{0}[\chi]-J \phi^{c} \\
& \quad+\int \frac{d \vec{k}}{(2 \pi)^{n-1}} \int_{t_{i}}^{t_{f}} d t\left(\dot{\phi}_{k}^{c} \dot{\chi}_{-k}-\omega_{k}^{2} \phi_{k}^{c} \chi_{-k}-j_{k}(t) \chi_{-k}(t)\right) .
\end{aligned}
$$

The last piece can be written as

$$
\begin{aligned}
\int_{t_{i}}^{t_{f}} d t & \left(\frac{d}{d t}\left(\dot{\phi}_{k}^{c} \chi_{-k}\right)-\chi_{-k} \ddot{\phi}_{k}^{c}-\omega_{k}^{2} \phi_{k}^{c} \chi_{-k}-j_{k}(t) \chi_{-k}(t)\right) \\
= & \dot{\phi}_{k}^{c}\left(t_{f}\right) \chi_{-k}\left(t_{f}\right)-\dot{\phi}_{k}^{c}\left(t_{i}\right) \chi_{-k}\left(t_{i}\right) \\
& -\int_{t_{i}}^{t_{f}} d t \chi_{-k}\left(\ddot{\phi}_{k}^{c}+\omega_{k}^{2} \phi_{k}^{c}+j_{k}(t)\right)
\end{aligned}
$$

and choose the classical field $\phi_{k}^{c}$ as the solution of the equation $\ddot{\phi}_{k}^{c}+\omega_{k}^{2} \phi_{k}^{c}+j_{k}(t)=0$, so

$$
\begin{aligned}
S_{0}[\phi]-J \phi= & S_{0}\left[\phi^{c}\right]-J \phi^{c}+S_{0}[\chi] \\
& +\left.\int d \vec{x}\left[\chi(x) \phi^{c}(x)\right]\right|_{t_{i}} ^{t_{f}} .
\end{aligned}
$$

There is an additional contribution coming from the wave functionals in the survival amplitude (2.1). For Fock vacuum wave functionals like (3.2), the exponent, depending on the boundary values of $\phi$, can be written as

$$
\begin{aligned}
& -\frac{1}{2} \int \frac{d \vec{k}}{(2 \pi)^{n-1}} \omega_{k}\left(\left|\varphi_{f k}\right|^{2}+\left|\varphi_{i k}\right|^{2}\right) \\
& =-\frac{1}{2} \int \frac{d \vec{k}}{(2 \pi)^{n-1}} \omega_{k}\left(\left|\phi_{k}^{c}\left(t_{i}\right)\right|^{2}+\left|\chi_{k}\left(t_{i}\right)\right|^{2}\right. \\
& \left.\quad+2 \chi_{-k}\left(t_{i}\right) \phi_{k}^{c}\left(t_{i}\right)+\left(t_{f} \text { term }\right)\right) .
\end{aligned}
$$

The full range of boundary terms in the sum of (3.6) and (3.7) is then

$$
\begin{aligned}
& i \dot{\phi}_{k}^{c}\left(t_{f}\right) \chi_{-k}\left(t_{f}\right)-i \dot{\phi}_{k}^{c}\left(t_{i}\right) \chi_{-k}\left(t_{i}\right)-\omega_{k}\left(\phi_{k}^{c}\left(t_{f}\right) \chi_{-k}\left(t_{f}\right)\right. \\
& \left.\quad+\chi_{-k}\left(t_{i}\right) \phi_{k}^{c}\left(t_{i}\right)\right),
\end{aligned}
$$

where the $i$ comes from the one in front of the action, $e^{i S_{0}}$. This means that if we impose on the classical solution $\phi^{c}$ the boundary conditions

$$
i \dot{\phi}_{k}^{c}\left(t_{f}\right)-\omega_{k} \phi_{k}^{c}\left(t_{f}\right)=0 i \dot{\phi}_{k}^{c}\left(t_{i}\right)+\omega_{k} \phi_{k}^{c}\left(t_{i}\right)=0
$$

these boundary terms vanish, and the classical field can be expressed in terms of the finite-time Feynman propagator, $\Delta_{T}\left(x, x^{\prime}\right)$, so that

$$
\phi^{c}(x) \equiv-\int d^{n} x^{\prime} \Delta_{T}\left(x, x^{\prime}\right) J\left(x^{\prime}\right) .
$$

Taking into account that

$$
\begin{aligned}
S_{0}\left[\phi^{c}\right]-J \phi^{c}= & \int d t d \vec{x} \frac{d}{d t}\left(\phi^{c} \dot{\phi}^{c}\right) \\
& -\int d^{n} x \frac{1}{2}\left(\square+m^{2}\right) \phi^{c}-J \phi^{c}= \\
= & \left.\frac{1}{2} \int d \vec{x}\left(\phi^{c} \dot{\phi}^{c}\right)\right|_{t_{i}} ^{t_{f}}-\frac{J \phi^{c}}{2} \\
= & -\int \frac{d \vec{k}}{(2 \pi)^{n-1}} \frac{\omega_{k}}{2} \\
& \times\left(\left|\phi_{k}^{c}\left(t_{f}\right)\right|^{2}+\left|\phi_{k}^{c}\left(t_{i}\right)\right|^{2}\right)-\frac{J \phi^{c}}{2}
\end{aligned}
$$

[where the boundary conditions obtained in Eq. (3.8) have been used], the first terms cancel precisely with the remaining $\left|\phi^{c}\right|^{2}$ contribution in (3.7). The full classical piece in the exponent is given by an expression quadratic ${ }^{2}$ in $J$

$$
\begin{aligned}
J \phi^{c} & \equiv \int_{t_{i}}^{t_{f}} d^{n} x J(x) \phi^{c}(x) \\
& =-\int_{t_{i}}^{t_{f}} d^{n} x \int_{t_{i}}^{t_{f}} d^{n} x^{\prime} J(x) J\left(x^{\prime}\right) \Delta_{T}\left(x, x^{\prime}\right) \equiv-J \Delta_{T} J
\end{aligned}
$$

and we are left with the following expression for the free survival amplitude:

$$
\left.\mathcal{A}_{0}\left(t_{f}, t_{i}\right)\right|_{J}=\left.e^{i / 2 \int_{t_{i}}^{t_{f}} d^{n} x d^{n} x^{\prime} J(x) \Delta_{T}\left(x, x^{\prime}\right) J\left(x^{\prime}\right)} \mathcal{A}_{0}\left(t_{f}, t_{i}\right)\right|_{J=0}
$$

We still have to compute $K_{0}[0]$ insofar as it depends on the initial and final times, $t_{i}$ and $t_{f}$, as well as the boundary conditions $\left[\varphi_{i}, \varphi_{f}\right]$, and integrate it convoluted with the remaining terms of the wave functionals. The exponents of these terms depend only on

\footnotetext{
${ }^{2}$ The time integration in the definition of $\phi^{c}$ takes place in the whole real line, while the time integration in the $J \phi^{c}$ term is constrained within the interval $\left[t_{i}, t_{f}\right]$. Nevertheless, the result can be proved to be independent of the value of the source $J$ outside this interval.
} 


$$
\left.\chi\right|_{t_{i}}=\varphi_{i}-\left.\phi^{c}\right|_{t_{i}},\left.\quad \chi\right|_{t_{f}}=\varphi_{f}-\left.\phi^{c}\right|_{t_{f}}
$$

so the integration variables can be shifted

$$
\left[D \varphi_{i}\right]\left[D \varphi_{f}\right]=\left[\left.D \chi\right|_{t_{i}}\right]\left[\left.D \chi\right|_{t_{f}}\right] .
$$

\section{Computation of the Schrödinger functional}

Let us compute the Schrödinger functional $K_{0}[0]\left[\chi_{i}, \chi_{f}\right]$ that is, the transition amplitude between states with welldefined values for the quantum fields in the free case. This is the quantity that in norelativistic quantum mechanics is aptly named Feynman's kernel

$$
K_{0}[0]\left[\chi^{f} t_{f}, \chi^{i} t_{i}\right] \equiv \int_{\chi^{i}}^{\chi^{f}} \mathcal{D} \chi e^{i S_{0}[\chi]} .
$$

This functional integral is computed with fixed Dirichlet boundary conditions at the endpoints and its functional form is

$$
K_{0}[0]\left[\chi^{f} t_{f}, \chi^{i} t_{i}\right]=e^{i S_{c}\left[\chi^{i}, \chi^{f}\right]} \operatorname{det}\left(\square+m^{2}\right)^{-(1 / 2)} .
$$

This expression can be achieved expanding again around a classical solution, given by

$$
\begin{aligned}
\chi(t, \vec{x})= & \int \frac{d \vec{k}}{(2 \pi)^{n-1}} \frac{\chi_{k}^{i} \sin \omega_{k}\left(t_{f}-t\right)+\chi_{k}^{f} \sin \omega_{k}\left(t-t_{i}\right)}{\sin \omega_{k} T} \\
& \times e^{-i \vec{k} \vec{x}}+\xi(t, \vec{x})
\end{aligned}
$$

so the classical action reads

$$
\begin{aligned}
S_{c}\left[\chi^{i}, \chi^{f}\right]= & \left.\frac{1}{2} \int \frac{d \vec{k}}{(2 \pi)^{n-1}} \chi_{k} \dot{\chi}_{-k}\right|_{t_{i}} ^{t_{f}} \\
= & \int \frac{d \vec{k}}{(2 \pi)^{n-1}} \frac{\omega_{k}}{2 \sin \omega_{k} T} \\
& \times\left[\left(\left|\chi_{k}^{i}\right|^{2}+\left|\chi_{k}^{f}\right|^{2}\right) \cos \omega_{k} T-2 \operatorname{Re} \chi_{k}^{i} \chi_{-k}^{f}\right]
\end{aligned}
$$

and the only thing that remains is to compute the determinant

$$
\operatorname{det}\left(\square+m^{2}\right) \equiv \int_{0}^{0} \mathcal{D} \xi e^{i S_{0}[\xi]}
$$

with vanishing boundary conditions $\left.\xi\right|_{t_{i}}=\left.\xi\right|_{t_{f}}=0$.

This determinant can be evaluated using zeta function regularization [14]. For even values of $n$, the corresponding zeta function is

$$
\begin{aligned}
\zeta^{\prime}(0)_{\mathrm{even}}= & \frac{\pi^{(n-1) / 2} m^{n-1}}{2(2 \pi)^{n-1}} V_{n-1}\left\{\frac{4(i m T)^{1-(n / 2)}}{\sqrt{\pi}} \sum_{j \geq 1} j^{-(n / 2)} K_{n / 2}(2 i m T j)-\Gamma\left(\frac{1-n}{2}\right)\right. \\
& \left.+\frac{(i m T)(-1)^{n / 2}}{\sqrt{\pi}(n / 2) !}\left[\gamma-\log \left(m^{2} / \mu^{2}\right)+\psi^{(0)}(1+n / 2)\right]\right\} .
\end{aligned}
$$

The sourceless Feynman kernel is precisely

$$
K_{0}[0]\left[\chi^{f}, \chi^{i}\right]=e^{i S_{c}} e^{-(1 / 2) \zeta^{\prime}(0)}
$$

\section{Flat space survival amplitude}

Let us study the vacuum survival amplitude in the free case performing the relevant integrals by brute force, i.e., without introducing any sources for the fields

$$
\begin{aligned}
\mathcal{A}_{0}\left(t_{f}, t_{i}\right) & \equiv\left\langle 0 t_{f} \mid 0 t_{i}\right\rangle=\int\left[D \varphi_{f}\right]\left[D \varphi_{i}\right]\left\langle 0 t_{f} \mid \varphi_{f} t_{f}\right\rangle\left\langle\varphi_{f} t_{f} \mid \varphi_{i} t_{i}\right\rangle\left\langle\varphi_{i} t_{i} \mid 0 t_{i}\right\rangle \\
& =|N|^{2} \int\left[D \varphi_{f}\right]\left[D \varphi_{i}\right] e^{-(1 / 2) \int \varphi_{f} \omega \varphi_{f}}\left\langle\varphi_{f} t_{f} \mid \varphi_{i} t_{i}\right\rangle e^{-(1 / 2) \int \varphi_{i} \omega \varphi_{i}},
\end{aligned}
$$

where we have introduced the vacuum wave functionals of Sec. III A, and the free Feynman kernel has just been shown to be

$$
K_{0}[0]\left[\varphi_{f} t_{f}, \varphi_{i} t_{i}\right] \equiv\left\langle\varphi_{f} t_{f} \mid \varphi_{i} t_{i}\right\rangle=\operatorname{det}\left(\square+m^{2}\right)^{-(1 / 2)} e^{i S_{c}\left[\varphi_{i}, \varphi_{f}\right]},
$$

where the determinant does not depend upon the boundary values for the field variables.

This yields

$\mathcal{S}_{0}\left(t_{f}, t_{i}\right)=|N|^{2} \operatorname{det}\left(+m^{2}\right)^{-(1 / 2)} \int\left[D \varphi_{f}\right]\left[D \varphi_{i}\right] \cdot \exp \left[i \int \frac{d \vec{k}}{(2 \pi)^{n-1}} \omega_{k}\left(\varphi_{f k}^{*} \varphi_{i k}^{*}\right)\left(\begin{array}{cc}\frac{i}{2}+\frac{1}{2} \cot \omega_{k} T & -\frac{1}{2 \sin \omega_{k} T} \\ -\frac{1}{2 \sin \omega_{k} T} & \frac{i}{2}+\frac{1}{2} \cot \omega_{k} T\end{array}\right)\left(\begin{array}{c}\varphi_{f k} \\ \varphi_{i k}\end{array}\right)\right]$.

We shall dub the functional determinant of the operator 


$$
B \equiv\left(\begin{array}{cc}
\frac{i}{2}+\frac{1}{2} \cot \omega_{k} T & -\frac{1}{2 \sin \omega_{k} T} \\
-\frac{1}{2 \sin \omega_{k} T} & \frac{i}{2}+\frac{1}{2} \cot \omega_{k} T
\end{array}\right)
$$

the boundary determinant.

The eigenvalues of this matrix are

$$
\lambda=-1-i \tan \left(\omega_{k} T / 2\right), \quad-1+i \cot \left(\omega_{k} T / 2\right)
$$

so the zeta function we have to consider in order to compute the boundary determinant is (recovering the $\omega_{k}$ factor we dropped in these eigenvalues)

$$
\begin{aligned}
\zeta(s) & =V_{n-1} \mu^{s} \int \frac{d \vec{k}}{(2 \pi)^{n-1}}\left(\frac{\omega_{k}}{2}\right)^{-s}\left\{\left(-\frac{e^{i \omega_{k} T / 2}}{\cos \omega_{k} T / 2}\right)^{-s}+\left(i \frac{e^{i \omega_{k} T / 2}}{\sin \omega_{k} T / 2}\right)^{-s}\right\} \\
& =\frac{V_{n-1} \mu^{s} \Omega_{n-2} m^{n-1-s}}{2^{-s}(2 \pi)^{n-1}} \int_{1}^{\infty} d x\left(x^{2}-1\right)^{(n-3) / 2} x^{1-s}\left\{\left(-\frac{e^{i x m T / 2}}{\cos x m T / 2}\right)^{-s}+\left(i \frac{e^{i x m T / 2}}{\sin x m T / 2}\right)^{-s}\right\} .
\end{aligned}
$$

In the even $n$ case the zeta function corresponding to the boundary determinant is

$$
\zeta^{\prime}(0)_{\mathrm{even}}=\frac{2 \Omega_{n-2} m^{n-1}}{(2 \pi)^{n-1}} \Gamma\left(\frac{n-1}{2}\right)\left\{\frac{1}{4} \Gamma\left(\frac{1-n}{2}\right)-\frac{(i m T)^{1-n / 2}}{2 \sqrt{\pi}} \sum_{j \geq 1} j^{-(n / 2)} K_{n / 2}(2 i m T j)\right\} .
$$

Collecting the results of this paragraph with the ones of the previous one, the vacuum survival amplitude in the even $n$ case reads

$$
\mathcal{A}_{0}\left(t_{f}, t_{i}\right) \equiv \exp \left[-\frac{1}{2} \frac{\pi^{(n-1) / 2} m^{n-1}}{2(2 \pi)^{n-1}} V_{n-1}\left\{\Gamma\left(\frac{1-n}{2}\right)+\frac{(i m T)(-1)^{n / 2}}{\sqrt{\pi}(n / 2) !}\left[\gamma-\log \left(m^{2} / 2 \mu^{2}\right)+\psi^{(0)}(1+n / 2)\right]\right\}\right] .
$$

In is remarkable that the end product of this computation is of the form

$$
\mathcal{A}_{0}\left(t_{f}, t_{i}\right) \propto e^{-i E_{0} T}
$$

with the vacuum energy given by

$$
\begin{aligned}
\frac{E_{0}}{V_{n-1}} \equiv & \frac{1}{2} \frac{\pi^{(n-1) / 2} m^{n}}{2(2 \pi)^{n-1}} \frac{(-1)^{n / 2}}{\sqrt{\pi}(n / 2) !} \\
& \times\left[\gamma-\log \left(m^{2} / 2 \mu^{2}\right)+\psi^{(0)}(1+n / 2)\right] .
\end{aligned}
$$

The inclusion of the interaction in these considerations can be achieved through Feynman diagrams with finitetime propagators built into them.

\section{SURVIVAL AMPLITUDES IN DE SITTER SPACE}

Let us now turn to the main object of our interest, namely, the (in)stability of the vacuum state in (anti) de Sitter space. We shall mainly use here the de Sitter metric in horospheric (Poincaré) coordinates, where $z$ plays the role of conformal time

$$
d s^{2}=\frac{l^{2} d z^{2}-\delta_{i j} d x^{i} d x^{j}}{z^{2}} .
$$

The conformal time is positive semidefinite

$$
0 \leq z \leq \infty
$$

It is sometimes useful to write $z \equiv e^{-H t}$, where the Hubble constant $H$ is related to the radius by $H \equiv \frac{1}{l}$, so that the metric appears in the steady state form

$$
d s^{2}=d t^{2}-e^{2 H t} \delta_{i j} d x^{i} d x^{j} .
$$

In these coordinates it is plain that in the limit $H \rightarrow 0$ $(l \rightarrow \infty)$ flat space is recovered.

We are interested in the survival amplitude of a certain state $\mid$ in $\rangle$ between (conformal) time $z$ and $z^{\prime}$ (both times can be finite)

$$
\begin{aligned}
\mathcal{A}_{\text {in }}\left(z_{f}, z_{i}\right) \equiv & \left.\left\langle\text { in } z_{f}\right| \text { in } z_{i}\right\rangle=\int\left[D \varphi_{f}\right]\left[D \varphi_{i}\right]\left\langle\text { in } z_{f} \mid \varphi_{f} z_{f}\right\rangle \\
& \left.\times\left\langle\varphi_{f} z_{f} \mid \varphi_{i} z_{i}\right\rangle\left\langle\varphi_{i} z_{i}\right| \text { in } z_{i}\right\rangle .
\end{aligned}
$$

\section{A. Wave functionals}

We shall expand the free field as

$$
\phi(z, \vec{x})=\int d \vec{p}\left(a_{p} v_{p}(z) e^{i \vec{p} \vec{x}}+a_{p}^{\dagger} v_{p}^{*}(z) e^{-i \vec{p} \vec{x}}\right)
$$

and the canonically conjugated momentum

$$
\pi(z, \vec{x})=\int \frac{d \vec{p}}{l z^{n-2}}\left(a_{p} v_{p}^{\prime}(z) e^{i \vec{p} \vec{x}}+a_{p}^{\dagger}\left(v_{p}^{\prime}\right)^{*}(z) e^{-i \vec{p} \vec{x}}\right) .
$$

Our modes are normalized by the usual Klein-Gordon invariant scalar product

$$
v_{p}^{\prime *} v_{p}-v_{p}^{*} v_{p}^{\prime}=i \frac{z^{n-2} l}{(2 \pi)^{n-1}} .
$$


The creation and annihilation operators are given by

$$
\begin{aligned}
a_{k}= & -i \frac{z^{2-n}}{l} \int d \vec{x} e^{-i \vec{k} \vec{x}} \\
& \times\left(\left(v_{k}^{\prime}\right)^{*}(x) \phi(z, \vec{x})-l z^{n-2} v_{k}^{*}(z) \pi(z, \vec{x})\right) \\
a_{k}^{\dagger}= & i \frac{z^{2-n}}{l} \int d \vec{x} e^{+i \vec{k} \vec{x}}\left(v_{k}^{\prime}(x) \phi(z, \vec{x})-l z^{n-2} v_{k}(z) \pi(z, \vec{x})\right) .
\end{aligned}
$$

It is now quite plain (at least formally) how to compute wave functions for different states. Let us begin with the wave function of the free (Fock) vacuum. It is defined for appropriate destruction operators and a given conformal time $z$ by

$$
\begin{aligned}
a_{k}|0\rangle & =\int d \vec{x} e^{-i \vec{k} \vec{x}}\left(v_{k}^{\prime}(z)^{*} \phi(z, \vec{x})-v_{k}(z)^{*} l z^{n-2} \pi(z, \vec{x})\right)|0\rangle \\
& =0 .
\end{aligned}
$$

We are thus led to a differential equation common for the vacuum wave function

$$
\int d \vec{x} e^{-i \vec{k} \vec{x}}\left(i v_{k}(z)^{*} z^{n-2} l \frac{\delta}{\delta \varphi(\vec{x})}+v_{k}^{\prime}(z)^{*} \varphi(\vec{x})\right)\langle\varphi z \mid 0\rangle=0 .
$$

The vacuum wave functional $\langle\varphi \mid 0\rangle$ is the exact analogue of the Schrödinger wave function $\psi(q, t) \equiv\langle q \mid \psi\rangle$, where the completeness relationship $\sum|q\rangle\langle q|=1$ is assumed in a time-independent way. Here we introduce a timeindependent basis $|\varphi\rangle$ such that

$$
\int[D \varphi]|\varphi\rangle\langle\varphi|=1
$$

This basis is defined in such a way that diagonalizes the field operator

$$
\hat{\phi}(\bar{z}, \vec{x})|\varphi\rangle=\varphi(\vec{x})|\varphi\rangle
$$

at a certain fiducial time, $\bar{z}$. But the basis itself depends on this fiducial time in a nontrivial way, and this we have attempted to represent by writing explicitly the basis as $\langle\varphi z|$. It follows that a Gaussian ansatz

$$
\langle\varphi z \mid 0\rangle=N e^{-(1 / 2) \int d \vec{x} d \vec{y} K_{z}(\vec{x}, \vec{y}) \varphi(\vec{x}) \varphi(\vec{y})}
$$

is indeed a solution, provided

$$
K_{z}(\vec{x}, \vec{y})=\frac{-i}{(2 \pi)^{n-1}} \int d \vec{p} e^{i \vec{p}(\vec{x}-\vec{y})} \frac{1}{l z^{n-2}} \frac{v_{p}^{\prime}(z)^{*}}{v_{p}(z)^{*}} .
$$

This gives a natural definition of noninteracting vacuum state corresponding to the modes $v_{p}(z)$. The present definition of vacuum depends on the modes used, and this in turn depends on the physical setup of the question asked. This is a general problem of quantum field theory in a curved space, not specific to our formalism.

Through the functional Schrödinger's equation, perturbative corrections to the noninteracting vacuum can easily be found. The concept of particle is a delicate one when asymptotically flat regions are absent. A possible definition of a multiparticle state in the present context is, for example,

$$
\left\langle\varphi z \mid k_{1} \ldots k_{p}\right\rangle \equiv\left\langle\varphi z\left|a_{k_{1}}^{\dagger} \ldots a_{k_{p}}^{\dagger}\right| 0\right\rangle
$$

but it is plain that the usefulness of such a definition is quite limited.

\section{B. Classical solutions}

The action for a scalar field in a generic conformally flat space (of which both de Sitter and anti-de Sitter are particular instances) can be written in a very simple form. We shall insist for no particular reason in keeping the coordinate $z$ dimensionless, so that the dimensionful coordinates are $x^{\mu} \equiv\left(x^{0}, x^{1}, \ldots x^{n-2}, l z\right)$. The metric is conformally flat

$$
d s^{2}=a(z)^{2} \eta_{\mu \nu} d x^{\mu} d x^{\nu} .
$$

In de Sitter space the coordinate $z$ is timelike, so that it follows that

$$
\begin{aligned}
S_{d S}[\phi]= & \int l d z d \vec{x} a^{n}\left(\frac{1}{a^{2}}\left(\frac{1}{l^{2}}\left(\partial_{z} \phi\right)^{2}-(\vec{\nabla} \phi)^{2}\right)\right. \\
& \left.-\frac{m^{2}}{2} \phi^{2}-\frac{\lambda}{4 !} \phi^{4}\right) .
\end{aligned}
$$

In anti-de Sitter, owing to the fact that the $z$ coordinate is spacelike, this reads

$$
\begin{aligned}
S_{A d S}[\phi]= & \int l d z d^{n-1} x a^{n}\left(\frac{1}{a^{2}}\left(-\frac{1}{l^{2}}\left(\partial_{z} \phi\right)^{2}+\dot{\phi}^{2}-(\vec{\nabla} \phi)^{2}\right)\right. \\
& \left.-\frac{m^{2}}{2} \phi^{2}-\frac{\lambda}{4 !} \phi^{4}\right)
\end{aligned}
$$

(where now $\vec{x}$ includes all coordinates except $x^{0} \equiv t$ and $z$ ).

We shall actually redefine the quantum field (but keep the same notation for it) in order to shift all dependence on the background towards the potential

$$
\phi_{\text {new }} \equiv a^{(n-2) / 2} \phi_{\text {old }}
$$

The Lagrangian now reads (remember, now $\phi \equiv \phi_{\text {new }}$ )

$$
\begin{aligned}
\mathcal{L}= & \frac{1}{2}(\partial \phi)^{2}-\frac{m(z)^{2}}{2} \phi^{2}-\frac{g(z)}{6} \phi^{3}-\frac{\lambda(z)}{24} \phi^{4} \\
& \pm \frac{2-n}{4 l^{2}} \frac{d}{d z}\left(\frac{\dot{a}}{a} \phi^{2}\right),
\end{aligned}
$$

where

$$
\begin{aligned}
m^{2}(z) & \equiv m^{2} a^{2} \pm\left(1-\frac{n}{2}\right) \frac{\ddot{a}}{a l^{2}} \mp\left(\frac{n}{2}-2\right)\left(\frac{n}{2}-1\right) \frac{\dot{a}^{2}}{a^{2} l^{2}} \lambda(z) \\
& \equiv a^{4-n} \lambda
\end{aligned}
$$

(where $\dot{a} \equiv \frac{d a}{d z}$; the upper signs are for de Sitter space, and the lower ones for anti-de Sitter).

In both de Sitter and anti-de Sitter, $a \equiv \frac{1}{z}$ which gives, paying due attention to the fact that $z$ is dimensionless, 


$$
\begin{aligned}
S_{d S}= & \frac{1}{2} \int d \vec{x} l d z\left\{\frac{1}{l^{2}}\left(\partial_{z} \phi\right)^{2}-(\nabla \phi)^{2}-\frac{m^{2} l^{2}-\frac{n(n-2)}{4}}{l^{2} z^{2}} \phi^{2}\right. \\
& \left.-\frac{\lambda}{12} \frac{z^{n-4}}{l} \phi^{4}\right\}+\left.\int d \vec{x} \frac{n-2}{4 z l} \phi^{2}\right|_{z_{i}} ^{z_{f}} \\
S_{A d S}= & \frac{1}{2} \int d^{n-1} x l d z\left\{-\frac{1}{l^{2}}\left(\partial_{z} \phi\right)^{2}+\dot{\phi}^{2}-(\nabla \phi)^{2}\right. \\
& \left.+\frac{m^{2} l^{2}+\frac{n(n-2)}{4}}{l^{2} z^{2}} \phi^{2}-\frac{\lambda}{12} \frac{z^{n-4}}{l} \phi^{4}\right\},
\end{aligned}
$$

where care has been taken to keep in de Sitter all boundary terms for future use. Incidentally, those are totally irrelevant for anti-de Sitter, because we are only integrating the time variable over a finite time interval; but they are quite important for de Sitter space, because they enforce a change in Feynman's propagator, as explained in the appendix in some detail.

It is amusing to remark that up to a constant factor the $\gamma$ factor defined in the appendix as $\gamma \equiv \frac{n-2}{2 z m l}$ is just the de Sitter temperature $T \equiv \frac{1}{4 \pi l}$

$$
\gamma m=\frac{n-2}{z} 2 \pi T .
$$

It is well-known that this temperature is associated with the unavoidable presence of a horizon because of the lack of a globally timelike Killing vector [11]. This coincidence is due to the fact that there is a single energy scale in de Sitter space.

This action can (and will) be interpreted as a Minkowskian action for a massive field, with a time-dependent potential given by

$$
\begin{aligned}
V(z, \phi) & \equiv-L_{I}(z, \phi) \\
& \equiv \frac{m^{2} l^{2}\left(1-z^{2}\right) \mp \frac{n(n-4)}{4}}{2 l^{2} z^{2}} \phi^{2}+\frac{\lambda}{24} z^{n-4} \phi^{4}
\end{aligned}
$$

The perturbation is a time-dependent one for de Sitter space, whereas it is space-dependent in anti-de Sitter. In this split between free and interacting Hamiltonian, all information on the curvature of the space has been dumped into the potential term.

\section{The Schrödinger functional}

The Schrödinger functional is given by finite-time Feynman's diagrams with position-dependent vertices

$$
\begin{aligned}
\left.\mathcal{A}\left(z_{f}, z_{i}\right)\right|_{J}= & e^{-i \int_{z_{i}}^{z_{f}} l d z d \vec{x} V(z, i(\delta / l \delta J))} \\
& \times\left.\left. e^{i / 2 \int_{z_{i}}^{z f} d^{n} x d^{n} x^{\prime} J(x) \Delta_{T}\left(x, x^{\prime}\right) J\left(x^{\prime}\right)}\right|_{J=0} \mathcal{A}_{0}\right|_{J=0} .
\end{aligned}
$$

To a given order in perturbation theory, it corresponds to vacuum diagrams (the same that contribute to the usual vacuum energy) computed with finite-time Feynman propagators. The important thing to notice is that the only dependence on the boundary values of the fields stems from the classical action.

The lowest-order diagrams contributing to this amplitude are depicted in Fig. 1. The first diagram to be computed is the "circle," which is simply

$$
M_{0,0}=\int_{z_{i}}^{z_{f}} l d z \int d \vec{x} \frac{d \vec{k}}{(2 \pi)^{n-1}} \Delta_{T}(k)[z, z]
$$

It is to be remarked that even this diagram carries some information about the curvature of the space through the $\gamma$ terms in the propagator.

We can take advantage of the specific form of the said propagator, in the sense that only the first coefficient (A13) contributes to the simple diagrams we will consider. In this case, the amplitude reads:

$$
M_{0,0}=V_{n-1} \int \frac{d \vec{k}}{(2 \pi)^{n-1}} \frac{e^{-2 i l \omega_{k} Z}\left(m\left(\gamma_{i}-\gamma_{f}\right)+e^{2 i l \omega_{k} Z}\left(4 l \omega_{k}^{2} Z+m\left(\gamma_{f}-\gamma_{i}\right)\left(1-2 i l \omega_{k} Z\right)\right)\right)}{4 \omega_{k}^{2}\left(m\left(\gamma_{i}-\gamma_{f}\right)-2 i \omega_{k}\right)}
$$

where we have neglected the product $\gamma_{i} \gamma_{f}$.

In the limits of large and small $Z$, (physically, the relevant quantity is $Z m l$ ) we have

$$
\begin{aligned}
M_{0,0} \stackrel{Z \rightarrow \infty}{\rightarrow} & \frac{V_{n-1} \Omega_{n-2} m^{n-3}}{2(2 \pi)^{n-1}}\left\{\operatorname{imlZJ}\left(\frac{n-3}{2}, 0\right)+\frac{\gamma_{i}}{2} I_{00}(Z)\right\} \\
M_{0,0} \stackrel{Z \rightarrow 0}{\rightarrow} & \frac{V_{n-1} \Omega_{n-2} m^{n-2}}{2(2 \pi)^{n-1}} \\
& \times\left\{i l Z J\left(\frac{n-3}{2}, 0\right)+\frac{\gamma_{i}}{2 z_{i}} l Z^{2} J\left(\frac{n-3}{2},-1\right)\right\}
\end{aligned}
$$

where

$$
\begin{aligned}
J(a, b) & =\int_{1}^{\infty} d x\left(x^{2}-1\right)^{a} x^{b} \\
I_{00}(Z) & =\int_{1}^{\infty} d x\left(x^{2}-1\right)^{(n-3) / 2} \frac{\left(e^{-2 i m l Z x}-1\right)}{\left(\gamma_{i}-2 i x\right) x} .
\end{aligned}
$$

The second diagram is the same as before, but with a mass insertion

$$
M_{1,0}=-\int_{z_{i}}^{z_{f}} l d z \int d \vec{x} \frac{d \vec{k}}{(2 \pi)^{n-1}} \Delta_{T}(k)[z, z]\left(\alpha+\frac{\beta}{z^{2}}\right)
$$

with $\alpha=-m^{2} / 2$ and $\beta=\left(m^{2}-n(n-2) / 4 l^{2}\right) / 2$. We have a part proportional to the first diagram, and a second part proportional to 


$$
\begin{aligned}
\int_{z_{i}}^{z_{f}} l d z & \frac{1}{2 z^{2}} e^{i l \omega_{k}\left(2 z^{\prime}-z_{f}-z_{i}\right)}\left(\left(2 \omega_{k}-i \gamma_{f} m\right) e^{2 i l \omega_{k}\left(z_{f}-z^{\prime}\right)}+i \gamma_{f} m\right)\left(\gamma_{i} m\left(-1+e^{2 i l \omega_{k}\left(z_{i}-z^{\prime}\right)}\right)+2 i \omega_{k}\right)= \\
= & \frac{1}{z_{f} z_{i}} l \omega_{k} e^{-i l \omega_{k}\left(z_{f}+z_{i}\right)}\left[-2 i l m \omega_{k} z_{f} z_{i}\left(\gamma_{f}\left(\operatorname{Ei}\left(2 i l z_{f} \omega_{k}\right)-\operatorname{Ei}\left(2 i l z_{i} \omega_{k}\right)\right)\right.\right. \\
& \left.\quad+\gamma_{i} e^{2 i l \omega_{k}\left(z_{f}+z_{i}\right)}\left(\operatorname{Ei}\left(-2 i l z_{f} \omega_{k}\right)-\operatorname{Ei}\left(-2 i l z_{i} \omega_{k}\right)\right)\right)-m e^{2 i l \omega_{k} z_{i}}\left(\gamma_{f} z_{f}+\gamma_{i} z_{i}\right) \\
& \left.\quad+e^{2 i l \omega_{k} z_{f}}\left(m\left(\gamma_{f} z_{f}+\gamma_{i} z_{i}\right)+2 i \omega_{k}\left(z_{f}-z_{i}\right)\right)\right],
\end{aligned}
$$

where we have neglected again the terms quadratic in the $\gamma$ 's.

For large $Z$ this contribution is just a constant independent of $Z$, while that for small $Z$ has a linear and a quadratic part. In this last limit, the full amplitude reads

$$
M_{1,0} \stackrel{Z \rightarrow 0}{\rightarrow}-\left(\alpha+\frac{\beta}{z_{i}^{2}}\right) \quad M_{00}+\frac{V_{n-1} \Omega_{n-2} m^{n-2}}{2(2 \pi)^{n-1} z_{i}^{3}} i l Z^{2} J\left(\frac{n-3}{2}, 0\right),
$$

where the $M_{00}$ has to be understood as the small- $Z$ limit shown above.

The third diagram (the first contribution of the self interaction) is given by

$$
M_{0,1}=3 i \int_{z_{i}}^{z_{f}} l d z \int d \vec{x} \frac{d \vec{k} d \vec{p}}{(2 \pi)^{2 n-2}} \Delta_{T}(k)[z, z] \Delta_{T}(p)[z, z] \lambda(z)
$$

with $\lambda(z)=z^{n-4} \lambda / 24$. The diagram is then proportional to

$$
\begin{array}{rl}
\int_{z_{i}}^{z_{f}} l & d z \frac{z^{n-4}}{4}\left(\left(2 \omega_{k}-i \gamma_{f} m\right) e^{2 i l \omega_{k}\left(z_{f}-z^{\prime}\right)}+i \gamma_{f} m\right)\left(\left(2 \omega_{p}-i \gamma_{f} m\right) e^{2 i l \omega_{p}\left(z_{f}-z^{\prime}\right)}+i \gamma_{f} m\right) \cdot\left(\gamma_{i} m\left(-1+e^{2 i l \omega_{k}\left(z_{i}-z^{\prime}\right)}\right)+2 i \omega_{k}\right) \\
& \times\left(\gamma_{i} m\left(-1+e^{2 i l \omega_{p}\left(z_{i}-z^{\prime}\right)}\right)+2 i \omega_{p}\right) e^{i l\left(\omega_{k}+\omega_{p}\right)\left(2 z^{\prime}-z_{f}-z_{i}\right)} \\
\simeq & -2 i l \omega_{k} \omega_{p} e^{-i l\left(\omega_{k}+\omega_{p}\right)\left(z_{f}+z_{i}\right)}\left[\gamma_{i} l^{4} m 2^{3-n} \omega_{k}^{4} \omega_{p}\left(i l \omega_{k}\right)^{-n-1} e^{2 i l\left(z_{f}\left(\omega_{k}+\omega_{p}\right)+\omega_{k} z_{i}\right)}\left(\Gamma\left(n-3,2 i l z_{f} \omega_{k}\right)-\Gamma\left(n-3,2 i l z_{i} \omega_{k}\right)\right)\right. \\
& +\gamma_{i} l^{4} m 2^{3-n} \omega_{k} \omega_{p}^{4}\left(i l \omega_{p}\right)^{-n-1} e^{2 i l\left(z_{f}\left(\omega_{k}+\omega_{p}\right)+\omega_{p} z_{i}\right)}\left(\Gamma\left(n-3,2 i l z_{f} \omega_{p}\right)-\Gamma\left(n-3,2 i l z_{i} \omega_{p}\right)\right) \\
& -\frac{1}{n-3}\left(z_{f}^{n-3}-z_{i}^{n-3}\right) e^{2 i l z_{f}\left(\omega_{k}+\omega_{p}\right)}\left(m\left(\gamma_{f}-\gamma_{i}\right)\left(\omega_{k}+\omega_{p}\right)+2 i \omega_{k} \omega_{p}\right) \\
& -\gamma_{f} m 2^{3-n} \omega_{p}\left(-i l \omega_{k}\right)^{3-n} e^{2 i l \omega_{p} z_{f}}\left(\Gamma\left(n-3,-2 i l z_{f} \omega_{k}\right)-\Gamma\left(n-3,-2 i l z_{i} \omega_{k}\right)\right) \\
& \left.-\gamma_{f} m 2^{3-n} \omega_{k}\left(-i l \omega_{p}\right)^{3-n} e^{2 i l \omega_{k} z_{f}}\left(\Gamma\left(n-3,-2 i l z_{f} \omega_{p}\right)-\Gamma\left(n-3,-2 i l z_{i} \omega_{p}\right)\right)\right] .
\end{array}
$$

For large $Z$, the leading term is proportional to $Z^{n-3}$

$$
\begin{aligned}
& M_{0,1} \stackrel{Z \rightarrow \infty}{\rightarrow} \frac{i \lambda V_{n-1} \Omega_{n-2}^{2} m^{2 n-4}}{32(2 \pi)^{2 n-2}}\left\{J\left(\frac{n-3}{2}\right)^{2} \frac{l Z^{n-3}}{n-3}+\left[-J\left(\frac{n-3}{2}, 0\right)^{2}+\frac{\gamma_{i}}{m l} I_{01}(Z)\right] l Z^{n-4}\right\} \\
& M_{0,1} \stackrel{Z \rightarrow 0}{\rightarrow} \frac{i \lambda \Omega_{n-2}^{2} V_{n-1} m^{2 n-4}}{32(2 \pi)^{2 n-2}} l J\left(\frac{n-3}{2}, 0\right)\left\{-z_{i}^{n-4} J\left(\frac{n-3}{2}, 0\right) Z+\frac{z_{i}^{n-5}}{2}\left[i \gamma_{i} J\left(\frac{n-3}{2},-1\right)-(n-4) J\left(\frac{n-3}{2}, 0\right)\right] Z^{2}\right\},
\end{aligned}
$$

where

$$
\begin{aligned}
I_{01}(Z)= & \int_{1}^{\infty} d x \int_{1}^{\infty} d y\left(x^{2}-1\right)^{(n-3) / 2}\left(y^{2}-1\right)^{(n-3) / 2} \\
& \times \frac{e^{-2 i m l Z x} y}{x\left(2 x y+\gamma_{i}(x+y)\right)} .
\end{aligned}
$$

\section{Survival amplitude}

The only step still left in order to compute the (vacuum) survival amplitude is the integration over the boundary values of the fields, weighed by the vacuum wave functions as well as the classical action. We know already from our previous computation (cf. Eq. (3.23)) that this contribution is subdominant in the large $Z$ limit, and besides it preserves the modulus of the (exponentiated) survival amplitude, so that it gives vanishing contribution to the width.

There are, however, calculable interaction dependent corrections to the vacuum wave function (as to any other wave function); they can be obtained through the functional Schrödinger's equation to any given order in 


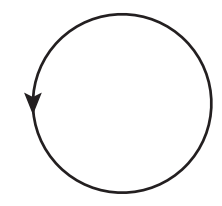

Order $(0,0)$

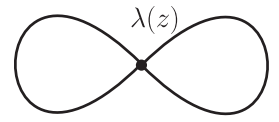

Order $(0,1)$

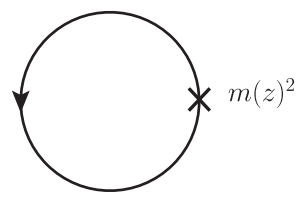

Order $(1,0)$

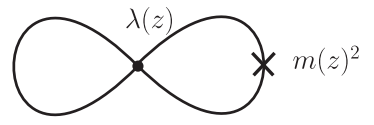

Order $(1,1)$
FIG. 1. The first few diagrams that contribute to the vacuum energy.

perturbation theory. We have not attempted to compute the effect of those corrections on the width.

In conclusion, the value we get for the width of the vacuum state in the asymptotic regime $Z \rightarrow \infty$ under the approximations of the present work is

$$
\begin{aligned}
\Gamma(Z) \stackrel{Z \rightarrow \infty}{\rightarrow} & \frac{\alpha V_{n-1} \Omega_{n-2} m^{n-3} \gamma_{i}}{2(2 \pi)^{n-1} Z} \operatorname{Re} I_{00}(Z) \\
& +\frac{\lambda V_{n-1} \Omega_{n-2}^{2} m^{2 n-5} \gamma_{i}}{16(2 \pi)^{2 n-2}} Z^{n-5} \operatorname{Im} I_{01}(Z)
\end{aligned}
$$

[Note added in proof.-It is possible to compute these integrals in the limit $\gamma_{i} \rightarrow 0$. Their asymptotic behavior is then

$$
I_{00}(Z) \rightarrow-\frac{i \Gamma\left(2-\frac{n}{2}\right) \Gamma\left(\frac{n-1}{2}\right)}{2 \sqrt{\pi}} I_{01}(Z) \rightarrow C_{n}(Z) Z^{(1-n) / 2}
$$

where $C_{n}$ is a pure oscillatory (bounded) function of $Z$. The asymptotic behavior of the survival amplitude in this limit is then proportional to $Z^{(n-9) / 2}$.]

\section{CONCLUSIONS}

In this paper we have concentrated in computing overlaps between arbitrary states (in particular the vacuum) defined at two different times such that they span a finite time interval (were this interval infinite they would become S-matrix elements, in case those happen to be welldefined). This has been done because there is some initial doubt as to how to define the good observables (i.e., as analogous to the decay rate, for example [2,4]) which would presumably involve some sort of square of the overlap matrix elements themselves.

The most important quantity we have analyzed is the survival rate, or self-overlap at a finite (conformal) time span. This in turn determines a decay width in a straightforward way. It is found that there some effects already at tree level, which are presumably related to particle creation in the presence of an external nonstatic gravitational field, but we have not identified them unambiguosly. Our computations are consistent with them being transients. They are however of potential physical relevance in the physics of the inflationary epoch.

At the next order in perturbation theory, there is a new contribution which determines the vacuum width in a precise way in the adequate space-time dimension [namely, $n=11$ were the dependence of both $I_{01}(Z)$ as well as $I_{00}(Z)$ on their argument subdominant]. Further diagrams should be studied before a definite conclusion can be drawn on the main issue.

This computation has been done for a particular wave function, which does receive corrections owing to the interaction. Other states can easily be studied within our framework. To the extent that flat space computations are a good guide, we do not expect those improvements to change the physical picture dramatically.

All the physical quantities studied in this paper turn out to be observer-dependent. It is not completely clear what could be the physical meaning of some phenomenon which is coordinate-dependent (or what amounts to more or less the same thing, observer-dependent). There are by now many examples of observer-dependent phenomena even in Minkowski space-time (of which the Unruh radiation [12] observed by an accelerated observer in the Minkowski vacuum is perhaps the best known); this does not necessarily mean that their physical meaning is fully understood.

On the other hand, it is well known that the usual semiclassical approximation to the full quantum theory of the gravitational field interacting with arbitrary matter, namely, quantum field theory in an external gravitational field treated classically (upon which the latter identification is based) is only an approximation to the true equations of motion, to wit,

$$
\left\langle\operatorname{vac}\left|\frac{\delta S}{\delta g^{\mu \nu}}\left[g_{\mu \nu}, \psi_{i}\right]\right| \mathrm{vac}\right\rangle=0 .
$$

Where the total action is the sum of the Einstein-Hilbert part depending on the metric only, the matter part, which dependS on the matter fields, denoted here collectively by $\psi_{i} i=1 \cdots N$, and the necessary counterterms, which depend on the metric as well as on the matter fields. Including sources,

$$
\begin{aligned}
S\left[J_{\mu \nu}, J_{i}\right] \equiv & S_{\mathrm{EH}}[g]+S_{\text {matt }}\left[g, \psi_{i}\right]+S_{\text {count }}\left[g, \psi_{i}\right] \\
& +i \int d^{n} x \sqrt{|g|}\left(J^{\mu \nu} g_{\mu \nu}+\sum_{i} J^{i} \psi_{i}\right) .
\end{aligned}
$$

The equations of motion are always formally true because they can be written in terms of the full partition function

$$
Z\left[J_{\mu \nu}, J_{i}\right] \equiv \int \mathcal{D} g_{\mu \nu} \prod_{i} \mathcal{D} \psi_{i} e^{i S\left[J_{\mu \nu}, J_{i}\right]}
$$

as

$$
\left.\frac{\delta S}{\delta g^{\mu \nu}}\left[\frac{1}{i} \frac{\delta}{\delta J_{\mu \nu}(x)}, \frac{1}{i} \frac{\delta}{\delta J_{i}(x)}\right] Z\left[J_{\mu \nu}, J_{i}\right]\right|_{J=0}=0 .
$$


It remains to give a working definition of the composite operator $g^{\mu \nu}$, but at the perturbative level this can be done. The state $|v a c\rangle$ is the one obtained through the boundary conditions imposed on the path integral.

The semiclassical framework states that this vacuum can be approximated by the matter vacuum in a fixed gravitational background $\bar{g}_{\mu \nu}$

$$
\mid \text { vac }\rangle \sim\left|0_{\text {matter }}\right\rangle_{\bar{g}} .
$$

This can be proven to be the dominant term the first term in a $1 / N$ expansion [13] of a theory of gravity interacting with $N$ identical matter species, but it is difficult to believe that this is the only instance in which this semiclassical approximation is physically reasonable. A general analysis of its validity would be welcome.

Observables in the full quantum gravity theory should presumably be gauge-invariant, that is, diffeomorphisminvariant, and thus independent on the observer. What seems to be needed here is a gauge-invariant definition of vacuum decay.

More comprehensive computations are in progress taking into account the dynamics of the gravity sector.

\section{ACKNOWLEDGMENTS}

This work has been partially supported by the European Commission (HPRN-CT-200-00148) as well as by FPA2009-09017 (DGI del MCyT, Spain) and S2009ESP1473 (CA Madrid). R. V. is supported by MEC Grant No. AP2006-01876.

\section{APPENDIX A: FINITE-TIME PROPAGATORS}

The general solution of the Klein-Gordon equation

$$
\left(\square+m^{2}\right) \phi=J
$$

can be written as

$$
\begin{aligned}
\phi(x)= & \int \frac{d \vec{k}}{(2 \pi)^{n-1}} e^{-i \vec{k} \vec{x}}\left(a_{k} \cos \omega_{k} t+b_{k} \sin \omega_{k} t\right) \\
& +\int d^{n} x^{\prime} \frac{d^{n} k}{(2 \pi)^{n}} e^{i k\left(x-x^{\prime}\right)} \frac{P}{-k_{0}^{2}+\omega_{k}^{2}} J\left(x^{\prime}\right) .
\end{aligned}
$$

The contribution of the principal value is

$$
\begin{aligned}
\int \frac{d k_{0}}{2 \pi} e^{i k_{0}\left(t-t^{\prime}\right)} \frac{P}{-k_{0}^{2}+\omega_{k}^{2}} \\
\quad=\int \frac{d k_{0}}{2 \pi} e^{i k_{0}\left(t-t^{\prime}\right)} \frac{1}{2 \omega_{k}}\left(\frac{P}{k_{0}+\omega_{k}}-\frac{P}{k_{0}-\omega_{k}}\right) \\
\quad=\frac{1}{2 \omega_{k}} S\left(t-t^{\prime}\right) \sin \omega_{k}\left(t-t^{\prime}\right),
\end{aligned}
$$

where we have used

$$
\begin{aligned}
\int d k \frac{P}{k-a} e^{i k x} & =\int d k \frac{e^{i k x}-e^{i k a}}{k-a}=e^{i k a} i \int d k \frac{\sin k x}{x} \\
& =e^{i k a} i \pi S(x)
\end{aligned}
$$

and $S(x) \equiv \theta(x)-\theta(-x)$ is the sign function. All this leads to

$$
\begin{aligned}
\phi(x)= & \int \frac{d \vec{k}}{(2 \pi)^{n-1}} e^{-i \vec{k} \vec{x}}\left(a_{k} \cos \omega_{k} t+b_{k} \sin \omega_{k} t\right) \\
& +\int d^{n} x^{\prime} \int \frac{d \vec{k}}{(2 \pi)^{n-1}} \frac{e^{-i \vec{k}\left(\vec{x}-\vec{x}^{\prime}\right)}}{2 \omega_{k}} \\
& \times S\left(t-t^{\prime}\right) \sin \omega_{k}\left(t-t^{\prime}\right) J\left(x^{\prime}\right) .
\end{aligned}
$$

In momentum space,

$$
\begin{aligned}
\phi_{-k}(t)= & a_{k} \cos \omega_{k} t+b_{k} \sin \omega_{k} t \\
& +\frac{1}{2 \omega_{k}} \int d^{n} x^{\prime} e^{i \vec{k} x^{\prime}} S\left(t-t^{\prime}\right) \sin \omega_{k}\left(t-t^{\prime}\right) J\left(x^{\prime}\right) .
\end{aligned}
$$

\section{Dirichlet boundary conditions}

The solution that vanishes at $t=t_{i}$ as well as at $t=t_{f}$ then reads

$$
\begin{aligned}
\phi(x)= & \int \frac{d \vec{k} d \vec{x}^{\prime} e^{-i \vec{k}\left(\vec{x}-\vec{x}^{\prime}\right)}}{(2 \pi)^{n-1} 2 \omega_{k} \sin \omega_{k}\left(t_{f}-t_{i}\right)} \int d t^{\prime} J\left(t^{\prime}, \vec{x}^{\prime}\right)\left[\sin \omega_{k}\left(t_{i}-t^{\prime}\right) S\left(t^{\prime}-t_{i}\right) \sin \omega_{k}\left(t_{f}-t\right)\right. \\
& \left.+\sin \omega_{k}\left(t_{f}-t^{\prime}\right) S\left(t^{\prime}-t_{f}\right) \sin \omega_{k}\left(t-t_{i}\right)+\sin \omega_{k}\left(t_{f}-t_{i}\right) \sin \omega_{k}\left(t-t^{\prime}\right) S\left(t-t^{\prime}\right)\right]
\end{aligned}
$$

It vanishes for $J=0$, in agreement with previous results.

This means that the correct propagator to be used in the integral over $\mathcal{D} \xi$ is given by

$$
\begin{aligned}
\mathcal{D}\left(x, x^{\prime}\right) \equiv & \int \frac{d \vec{k} e^{-i \vec{k}\left(\vec{x}-\vec{x}^{\prime}\right)}}{2(2 \pi)^{n-1} \omega_{k} \sin \omega_{k} T}\left[\sin \omega_{k}\left(t_{i}-t^{\prime}\right) S\left(t^{\prime}-t_{i}\right) \sin \omega_{k}\left(t_{f}-t\right)+\sin \omega_{k}\left(t_{f}-t^{\prime}\right) S\left(t^{\prime}-t_{f}\right) \sin \omega_{k}\left(t-t_{i}\right)\right. \\
& \left.+\sin \omega_{k} T \sin \omega_{k}\left(t-t^{\prime}\right) S\left(t-t^{\prime}\right)\right] .
\end{aligned}
$$


That is, this is the only solution to the equation

$$
\left(\square+m^{2}\right) \mathcal{D}\left(x, x^{\prime}\right)=\delta\left(x-x^{\prime}\right)
$$

such that

$$
\left.\mathcal{D}\left(x, x^{\prime}\right)\right|_{t=t_{i}}=\left.\mathcal{D}\left(x, x^{\prime}\right)\right|_{t^{\prime}=t_{f}}=0
$$

\section{Feynman boundary conditions}

The boundary conditions for the Feynman propagator are defined by

$$
i \dot{\phi}_{k}\left(t_{f}\right)=\omega_{k} \phi_{k}\left(t_{f}\right) \quad i \dot{\phi}_{k}\left(t_{i}\right)=-\omega_{k} \phi_{k}\left(t_{i}\right) .
$$

In momentum space

$$
\begin{aligned}
\dot{\phi}_{-k}(t)= & -\omega_{k} a_{k} \sin \omega_{k} t+b_{k} \omega_{k} \cos \omega_{k} t \\
& +\int_{\mathbb{R}^{n}} d^{n} x^{\prime} \frac{e^{i \vec{k} \vec{x}^{\prime}}}{2 \omega_{k}} S\left(t-t^{\prime}\right) \omega_{k} \cos \omega_{k}\left(t-t^{\prime}\right) J\left(x^{\prime}\right)
\end{aligned}
$$

(the delta function does not contribute).

The boundary conditions are then

$$
\begin{gathered}
-i \omega_{k} a_{k} \sin \omega_{k} t_{f}+i b_{k} \omega_{k} \cos \omega_{k} t_{f}+i \int_{\mathbb{R}^{n}} d^{n} x^{\prime} \frac{e^{i \vec{k} \vec{x}^{\prime}}}{2 \omega_{k}} S\left(t_{f}-t^{\prime}\right) \omega_{k} \cos \omega_{k}\left(t_{f}-t^{\prime}\right) J\left(x^{\prime}\right) \\
=\omega_{k}\left(a_{k} \cos \omega_{k} t_{f}+b_{k} \sin \omega_{k} t_{f}+\frac{1}{2 \omega_{k}} \int_{\mathbb{R}^{n}} d^{4} x^{\prime} e^{i \vec{k} \vec{x}^{\prime}} S\left(t_{f}-t^{\prime}\right) \sin \omega_{k}\left(t_{f}-t^{\prime}\right) J\left(x^{\prime}\right)\right) \\
-i \omega_{k} a_{k} \sin \omega_{k} t_{i}+i b_{k} \omega_{k} \cos \omega_{k} t_{i}+i \int_{\mathbb{R}^{n}} d^{n} x^{\prime} \frac{e^{i \overrightarrow{x^{\prime}}}}{2 \omega_{k}} S\left(t_{i}-t^{\prime}\right) \omega_{k} \cos \omega_{k}\left(t_{i}-t^{\prime}\right) J\left(x^{\prime}\right) \\
=-\omega_{k}\left(a_{k} \cos \omega_{k} t_{i}+b_{k} \sin \omega_{k} t_{i}+\frac{1}{2 \omega_{k}} \int_{\mathbb{R}^{n}} d^{4} x^{\prime} e^{i \vec{k} \vec{x}^{\prime}} S\left(t_{i}-t^{\prime}\right) \sin \omega_{k}\left(t_{i}-t^{\prime}\right) J\left(x^{\prime}\right)\right) .
\end{gathered}
$$

This can be written as

$$
\left(\begin{array}{cc}
-e^{i \omega_{k} t_{f}} & i e^{i \omega_{k} t_{f}} \\
e^{-i \omega_{k} t_{i}} & i e^{-i \omega_{k} t_{i}}
\end{array}\right)\left(\begin{array}{c}
a_{k} \\
b_{k}
\end{array}\right)=-\frac{1}{2 \omega_{k}} \int_{\mathbb{B}^{n}} d^{n} x^{\prime} e^{i \vec{k} \vec{x}^{\prime}}\left(\begin{array}{c}
S\left(t_{f}-t^{\prime}\right) i e^{i \omega_{k}\left(t_{f}-t^{\prime}\right)} \\
S\left(t_{i}-t^{\prime}\right) i e^{-i \omega_{k}\left(t_{i}-t^{\prime}\right)}
\end{array}\right) J\left(x^{\prime}\right)
$$

and this yields

$$
\begin{aligned}
a_{k} & =\frac{i}{4 \omega_{k}} e^{-i \omega_{k} T} \int d^{n} x^{\prime} e^{i \vec{k} \vec{x}^{\prime}} J\left(x^{\prime}\right)\left(S\left(t_{f}-t^{\prime}\right) e^{-i \omega_{k}\left(t^{\prime}-T\right)}-S\left(t_{i}-t^{\prime}\right) e^{i \omega_{k}\left(T+t^{\prime}\right)}\right) \\
& =\frac{i}{4 \omega_{k}} \int_{\mathbb{R}^{n}} d^{n} x^{\prime} e^{i \vec{k} \vec{x}^{\prime}} J\left(x^{\prime}\right)\left(S\left(t_{f}-t^{\prime}\right) e^{-i \omega_{k} t^{\prime}}-S\left(t_{i}-t^{\prime}\right) e^{\left.i \omega_{k} t^{\prime}\right),}\right. \\
b_{k} & =-\frac{1}{4 \omega_{k}} \int_{\mathbb{R}^{n}} d^{n} x^{\prime} e^{i \vec{k} \vec{x}^{\prime}} J\left(x^{\prime}\right) e^{-i \omega_{k} T}\left(S\left(t_{f}-t^{\prime}\right) e^{i \omega_{k}\left(T-t^{\prime}\right)}+S\left(t_{i}-t^{\prime}\right) e^{i \omega_{k}\left(T+t^{\prime}\right)}\right) \\
& =-\frac{1}{4 \omega_{k}} \int_{\mathbb{R}^{n}} d^{n} x^{\prime} e^{i \vec{k} \vec{x}^{\prime}} J\left(x^{\prime}\right)\left(S\left(t_{f}-t^{\prime}\right) e^{-i \omega_{k} t^{\prime}}+S\left(t_{i}-t^{\prime}\right) e^{\left.i \omega_{k} t^{\prime}\right) .}\right.
\end{aligned}
$$

This means that the corresponding classical solution reads

$$
\phi(x) \equiv \int_{\mathbb{R}^{n}} d^{n} x^{\prime} \Delta_{T}\left(x, x^{\prime}\right) J\left(x^{\prime}\right)
$$

with $\Delta_{T}$ the finite-time Feynman propagator

$$
\begin{aligned}
\Delta_{T}\left(x, x^{\prime}\right) \equiv & \int \frac{d \vec{k}}{(2 \pi)^{n-1}} e^{-i \vec{k}\left(\vec{x}-\vec{x}^{\prime}\right)} \\
\Delta_{T}(k) \Delta_{T}(k)= & \frac{i}{4 \omega_{k}}\left[\left(S\left(t_{f}-t^{\prime}\right) e^{-i \omega_{k} t^{\prime}}-S\left(t_{i}-t^{\prime}\right) e^{i \omega_{k} t^{\prime}}\right) \cos \omega_{k} t+i\left(S\left(t_{f}-t^{\prime}\right) e^{-i \omega_{k} t^{\prime}}+S\left(t_{i}-t^{\prime}\right) e^{i \omega_{k} t^{\prime}}\right) \sin \omega_{k} t\right] \\
& +\frac{1}{2 \omega_{k}} S\left(t-t^{\prime}\right) \sin \omega_{k}\left(t-t^{\prime}\right) .
\end{aligned}
$$


It is then plain that in the limit $t_{f}=-t_{i}=\frac{T}{2}$ and $T \rightarrow \infty$, Feynman's continuum result is recovered.

$$
\begin{aligned}
\lim _{T \rightarrow \infty} \Delta_{T}(k) & =\Delta_{F}(k) \\
& \equiv \frac{i}{2 \omega_{k}}\left[\cos \omega_{k}\left(t-t^{\prime}\right)-i S\left(t-t^{\prime}\right) \sin \omega_{k}\left(t-t^{\prime}\right)\right]
\end{aligned}
$$

In general it yields

$$
\begin{aligned}
\Delta_{T}(k)= & \frac{i}{4 \omega_{k}}\left(\left[S\left(t_{f}-t^{\prime}\right)-S\left(t_{i}-t^{\prime}\right)-2\right] \cos \omega_{k}\left(t-t^{\prime}\right)\right. \\
& \left.+i\left[S\left(t_{f}-t^{\prime}\right)+S\left(t_{i}-t^{\prime}\right)\right] \sin \omega_{k}\left(t-t^{\prime}\right)\right)+\Delta_{F}(k) .
\end{aligned}
$$

The above results are valid for general sources with arbitrary support. When (as in our case) the support is restricted to the interval $t_{i} \leq t^{\prime} \leq t_{f}$, it is quite easy to check that the finite-time propagator with Feynman's boundary conditions coincides exactly with one usual Feynman's propagator.

$$
\left.\Delta_{T}(k)\right|_{\left[t_{i}, t_{f}\right]}=\left.\Delta_{F}(k)\right|_{\left[t_{i}, t_{f}\right]} .
$$

\section{Feynman's propagator including de Sitter boundary terms}

The boundary terns that appear when redefining the field in de Sitter space imply (after the splitting $\phi=\phi_{c}+\chi$ ) an addition of

$$
\begin{aligned}
& i \frac{n-2}{2 z_{f} l} \phi_{c}\left(z_{f}\right) \chi\left(z_{f}\right)-i \frac{n-2}{2 z_{i} l} \phi_{c}\left(z_{i}\right) \chi\left(z_{i}\right) \\
& \equiv i \gamma_{f} m \phi_{c}\left(z_{f}\right) \chi\left(z_{f}\right)-i \gamma_{i} m \phi_{c}\left(z_{i}\right) \chi\left(z_{i}\right)
\end{aligned}
$$

to the boundary. In order to eliminate those cross-terms the boundary conditions to be imposed are

$$
\begin{aligned}
i \dot{\phi}_{k}^{c}\left(z_{f}\right)-\omega_{k} l \phi_{k}^{c}\left(z_{f}\right)+i \gamma_{f} m l \phi_{k}^{c}\left(z_{f}\right) & =0 \\
i \dot{\phi}_{k}^{c}\left(z_{i}\right)+\omega_{k} l \phi_{k}^{c}\left(z_{i}\right)+i \gamma_{i} m l \phi_{k}^{c}\left(z_{i}\right) & =0 .
\end{aligned}
$$

Let us now make a slightly different antsatz for the form of the propagator, namely

$$
\begin{aligned}
\Delta_{T}(k)= & \frac{1}{2 \omega_{k}} S\left(z-z^{\prime}\right) \sin \omega_{k} l\left(z-z^{\prime}\right) \\
& +a_{k} \cos \omega_{k} l\left(z-z^{\prime}\right)+b_{k} \sin \omega_{k} l\left(z-z^{\prime}\right) .
\end{aligned}
$$

The previous equations for the boundary values give rise to the following coefficients:

$$
\begin{aligned}
D_{k} \equiv & \frac{e^{-i l \omega_{k} Z}}{\omega_{k}\left(-\gamma_{f} \gamma_{i} m^{2}\left(e^{2 i l \omega_{k} Z}-1\right)-2 i m \omega_{k}\left(\gamma_{f}-\gamma_{i}\right)+4 \omega_{k}^{2}\right)} \\
a_{k}= & \frac{D_{k}}{4}\left(\left(2 \omega_{k}-i \gamma_{f} m\right) e^{2 i l \omega_{k}\left(z_{f}-z^{\prime}\right)}+i \gamma_{f} m\right)\left(\gamma_{i} m\left(-1+e^{2 i l \omega_{k}\left(z_{i}-z^{\prime}\right)}\right)+2 i \omega_{k}\right) \cdot\left(S\left(z_{f}-z^{\prime}\right)-S\left(z_{i}-z^{\prime}\right)\right) e^{i l \omega_{k}\left(2 z^{\prime}-z_{f}-z_{i}\right)} \\
b_{k}= & \frac{D_{k}}{4} e^{-i l \omega_{k}\left(2 z^{\prime}+z_{f}+z_{i}\right)}\left[S\left(z_{f}-z^{\prime}\right)\left(\gamma_{f} m e^{2 i l z^{\prime} \omega_{k}}+e^{2 i l \omega_{k} z_{f}}\left(\gamma_{f}(-m)-2 i \omega_{k}\right)\right)\left(e^{2 i l z^{\prime} \omega_{k}}\left(\gamma_{i} m-2 i \omega_{k}\right)+\gamma_{i} m e^{2 i l \omega_{k} z_{i}}\right)\right. \\
& \left.+S\left(z^{\prime}-z_{i}\right)\left(\gamma_{f} m e^{2 i l z^{\prime} \omega_{k}}+e^{2 i l \omega_{k} z_{f}}\left(\gamma_{f} m+2 i \omega_{k}\right)\right) \cdot\left(e^{2 i l z^{\prime} \omega_{k}}\left(\gamma_{i} m-2 i \omega_{k}\right)-\gamma_{i} m e^{2 i l \omega_{k} z_{i}}\right)\right],
\end{aligned}
$$

where $Z=z_{f}-z_{i}$.

However, the interaction takes place only in the interval $\left[z_{i}, z_{f}\right]$, so we should restrict the variables $z$ and $z^{\prime}$ to be in this interval, so

$$
\begin{aligned}
& a_{k}=\frac{D_{k}}{2} e^{i l \omega_{k}\left(2 z^{\prime}-z_{f}-z_{i}\right)}\left(\left(2 \omega_{k}-i \gamma_{f} m\right) e^{2 i l \omega_{k}\left(z_{f}-z^{\prime}\right)}+i \gamma_{f} m\right)\left(\gamma_{i} m\left(-1+e^{2 i l \omega_{k}\left(z_{i}-z^{\prime}\right)}\right)+2 i \omega_{k}\right) \\
& b_{k}=\frac{D_{k}}{2} m e^{-i l \omega_{k}\left(2 z^{\prime}+z_{f}+z_{i}\right)}\left(\gamma_{f} e^{4 i l z^{\prime} \omega_{k}}\left(\gamma_{i} m-2 i \omega_{k}\right)-\gamma_{i}\left(\gamma_{f} m+2 i \omega_{k}\right) e^{2 i l \omega_{k}\left(z_{f}+z_{i}\right)}\right) .
\end{aligned}
$$

Also, since the exponent of the Feynman kernel is symmetric in the source $J$, we must symmetrize the propagator.

[1] A. M. Polyakov, Nucl. Phys. B797, 199 (2008); Nucl. Phys. B834, 316 (2010).

[2] E. Alvarez and R. Vidal, J. High Energy Phys. 10 (2009) 045; J. Cosmol. Astropart. Phys. 11 (2010) 043.

[3] A. Higuchi, Classical Quantum Gravity 26, 072001 (2009).
[4] J. Bros, H. Epstein, and U. Moschella, Ann. Inst. Henri Poincaré 11, 611 (2010); J. Cosmol. Astropart. Phys. 02 (2008) 003.

[5] S. Weinberg, The Quantum Theory of Fields (Cambridge University Press, Cambridge, England, 1995).

[6] L. Maiani and M. Testa, Ann. Phys. (N.Y.) 263, 353 (1998). 
[7] J. J. Sakurai, Advanced Quantum Mechanics (Addison Wesley, Reading, MA, 1967); Modern Quantum Mechanics (Addison Wesley, Reading, MA, 1994).

[8] R. Jackiw, in Diverse Topics in Theoretical and Mathematical Physics, edited by R. Jackiw (World Scientific, Singapore, 1995), pp. 383-445.

[9] D. V. Long and G. M. Shore, Nucl. Phys. B530, 247 (1998); Nucl. Phys. B530, 279 (1998).
[10] B. Sakita, Quantum Theory of Many-Variable Systems and Elds (World Scientific, Singapore, 1985).

[11] G. W. Gibbons and S. W. Hawking, Phys. Rev. D 15, 2738 (1977).

[12] W. G. Unruh, Phys. Rev. D 14, 870 (1976).

[13] J. B. Hartle and G. T. Horowitz, Phys. Rev. D 24, 257 (1981).

[14] E. Alvarez and A.F. Faedo, J. High Energy Phys. 05 (2006) 046. 